\title{
Articulación de saberes y sabores contra la pérdida y despilfarro alimentario en América Latina y El Caribe
}

\section{Articulation of knowledge and flavors against food loss and waste in Latin America and the Caribbean}

Articulação de saberes e sabores contra a perda e o desperdício de alimentos na América Latina e no Caribe

\author{
Adriana Arroyo ${ }^{1}$ \\ Universidad El Bosque, Bogotá - Bogotá, Colombia \\ (iD https://orcid.org/0000-0002-3577-292X \\ arroyoadriana@unbosque.edu.co
}

DOI: https://doi.org/10.35622/j.rg.2022.01.002

Recibido: 26/10/2021 Aceptado: 28/01/2022 Publicado: 28/01/2022

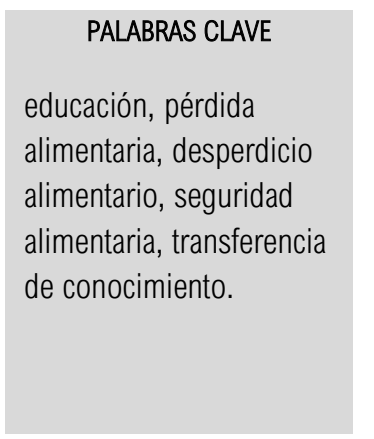

KEYWORDS
education, food loss,
food waste, food
security, knowledge
transfer.

PALAVRAS-CHAVE

educação, perda de alimentos, desperdício

\begin{abstract}
RESUMEN. Las cifras de inseguridad alimentaria son alarmantes en América Latina y El Caribe, así como, los porcentajes de pérdida y desperdicio alimentario, con sus consiguientes pérdidas de energía, agua potable y desde luego de dinero. Frente a este panorama, el presente artículo de reflexión, se realizó desde un enfoque académico. Se recurrió a la revisión de fuentes originales, para ofrecer una mirada analítica, sobre las acciones decididas y articuladas, entre academia y empresa. Se encontró que es primordial articular esfuerzos y acciones efectivas tanto desde el gobierno, el sector privado como en la población, que estén encausados a desarrollar una visión y soluciones comunes como la dupla garante de soluciones sostenibles. Esto da paso hacia la creación de empleo, la generación de valor en las cadenas de suministro alimentario, la merma de la pérdida y desperdicio alimentario a nivel global.
\end{abstract}

\begin{abstract}
Food insecurity figures are alarming in Latin America and the Caribbean and the percentages of food loss and waste, with consequent energy losses, drinking water, and of course, money. Faced with this panorama, this reflection article was conducted academically. A review of sources was used to offer an analytical view of academia and businesses decided and articulated actions. It was found that it is essential to say efforts and practical activities both from the government, the private sector, and the population, which aim to develop a shared vision and solutions as the guarantor of sustainable solutions. This gives way to the creation of employment, the generation of value in food supply chains, the reduction of food loss and waste globally.
\end{abstract}

RESUM0. Os números da insegurança alimentar são alarmantes na América Latina e no Caribe, assim como os percentuais de perda e desperdício de alimentos, com suas consequentes perdas de energia, água potável e, claro, dinheiro. Diante desse panorama, este artigo de reflexão foi realizado a partir de uma abordagem acadêmica. Uma revisão de fontes originais foi usada para

1Docente en la Universidad El Bosque. Correspondencia: arroyoadriana@unbosque.edu.co 
de alimentos, segurança alimentar, transferência de conhecimento. oferecer uma visão analítica das ações decididas e articuladas entre academia e empresa. Constatou-se que é fundamental articular esforços e ações efetivas tanto do governo, da iniciativa privada e da população, que visem o desenvolvimento de uma visão e soluções comuns como garantia de soluções sustentáveis. Isso dá lugar à criação de empregos, à geração de valor nas cadeias de abastecimento de alimentos, à redução da perda e do desperdício de alimentos globalmente.

\section{INTRODUCCIÓN}

A pesar de los avances que se han hecho en América Latina y El Caribe, especialmente en la revisión y monitoreo de implementación de marcos normativos, para fortalecer la seguridad alimentaria y la optimización de los sistemas alimentarios, las medidas y resultados siguen siendo insuficientes para cumplir con los indicadores plenos de los Objetivo de Desarrollo Sostenible 2 y 12 , entre otros.

En la región se pierde el $11,6 \%$ de los alimentos, desde la producción hasta el comercio minorista. Esto equivale a 220 millones de toneladas al año con un costo económico de 150.000 millones de dólares (Bélanger \& Pilling, 2019). En países en vía de desarrollo, prevalecen las pérdidas de alimentos en los segmentos iniciales e intermedios de la cadena de valor, debido a la deficiencia en el acceso a insumos, pero principalmente por mal estado de las carreteras, cierres y manifestaciones sociales y una logística deficiente en los centros de acopio y transformación.

Los retos para los gobiernos y las poblaciones del continente son cada vez más desafiantes, debido a las crecientes amenazas para preservar la biodiversidad como factor clave para los sistemas alimentarios (FA0, 2020b). El informe de la Organización de las Naciones Unidas para la Alimentación y la Agricultura sobre biodiversidad para la alimentación y la agricultura en el mundo, pone en el punto de mira la imparable degradación de la biodiversidad y por ende los medios de subsistencia (Grisa \& Sabourin, 2019). Menos

biodiversidad significa que las plantas y los animales sean más vulnerables a plagas y enfermedades. La pérdida creciente de biodiversidad para la alimentación y la agricultura, agravada por nuestra dependencia de cada vez menos especies para alimentarnos, lleva nuestra ya frágil seguridad alimentaria al borde del colapso (FAO, 2020a).

Este trabajo examina brevemente, desde la labor de revisión del autor, los retos puntuales que tiene el fortalecimiento de los sistemas alimentarios de la región, antes de la pandemia de la Covid 19 y acrecentados por la misma, centrándose en la pérdida de alimentos y recoge diferentes acciones que vienen implementando gobiernos, entidades educativas e instituciones privadas, para paliar esta situación.

\section{MÉTODO Y MATERIALES}

Este artículo de revisión recoge de forma sistematizada, la conceptualización de literatura científica sobre el tema de la pérdida de alimentos destinados al consumo humano, realizada por organismos internacionales como la Organización de las Naciones Unidas para la Alimentación (FA0), Programa de las Naciones Unidas para el Desarrollo (PNUD) y la Comunidad de Estados Latinoamericanos y Caribeños (CELAC). Teniendo en cuenta que los indicadores y estadísticas sobre este particular son escasos y evidencian actualizaciones parciales. 
La estructuración del presente trabajo, formula consideraciones para alentar futuras revisiones sobre la implementación de buenas prácticas, escalables y replicables en todo el continente.

\section{RESULTADOS}

\section{Perspectivas de sistemas alimentarios en el continente latino americano}

En cada una de las etapas del sistema alimentario desarrollados en América Latina, como lo son la producción, elaboración, distribución, preparación y consumo de alimentos, se evidencian porcentajes de pérdida, desperdicio alimentario, un malgasto de energía, insumos, agua potable y mano de obra, además de reprocesos para la gestión eficiente de residuos orgánicos con el consiguiente impacto ambiental. Esto supone, sin lugar a duda, un gasto innecesario en cuanto a lo económico y energético.

A esta considerable pérdida de alimentos producidos para el consumo humano, se suma la desigualdad nutricional, la cual se relaciona con regiones menos desarrolladas. En países de renta media baja de América Latina y El Caribe, se evidencia un acceso limitado a alimentos frescos y saludables. Esto como consecuencia de políticas agrarias deficientes, producción, distribución inadecuadas y estrategias de precios desfavorecedoras para estas poblaciones. Se tiene como consecuencia que más de la mitad de ciudadanía, no puede acceder a una dieta saludable.

Antes de la pandemia de la Covid 19, las medidas para minimizar la pérdida y desperdicio de alimentos, así como para robustecer la seguridad alimentaria que lideraban a nivel gobierno, se enfocaban en garantizar mejoras en ambos aspectos, mediante el fortalecimiento de las primeras etapas del sistema agroalimentario, especialmente en las explotaciones agrícolas en países de menor ingreso de América Latina y El Caribe. Desde el año 2019, las debilidades e inequidades ya existentes en estos sistemas agroalimentarios, se han recrudecido como consecuencia de las interrupciones prolongadas en el funcionamiento de la logística, el cierre de puertos (aéreos y marítimos), las restricciones en los desplazamientos y las medidas de bioseguridad, como medidas para mitigar la propagación del virus.

De la misma forma se ha visto altamente perjudicado, el nivel de empleabilidad y su consiguiente falta de ingresos económicos en cada familia del continente, debido a la emergencia sanitaria por el Covid-19. Los impactos sobre la oferta y demanda de alimentos dependerán de las estructuras productivas y comerciales de los países, de sus niveles y grado de desigualdad de los ingresos, y de factores externos relacionados con los mercados (FA0, 2020a). 


\section{Figura 1.}

Pérdida de alimentos e Inseguridad alimentaria antes y después de la pandemia.

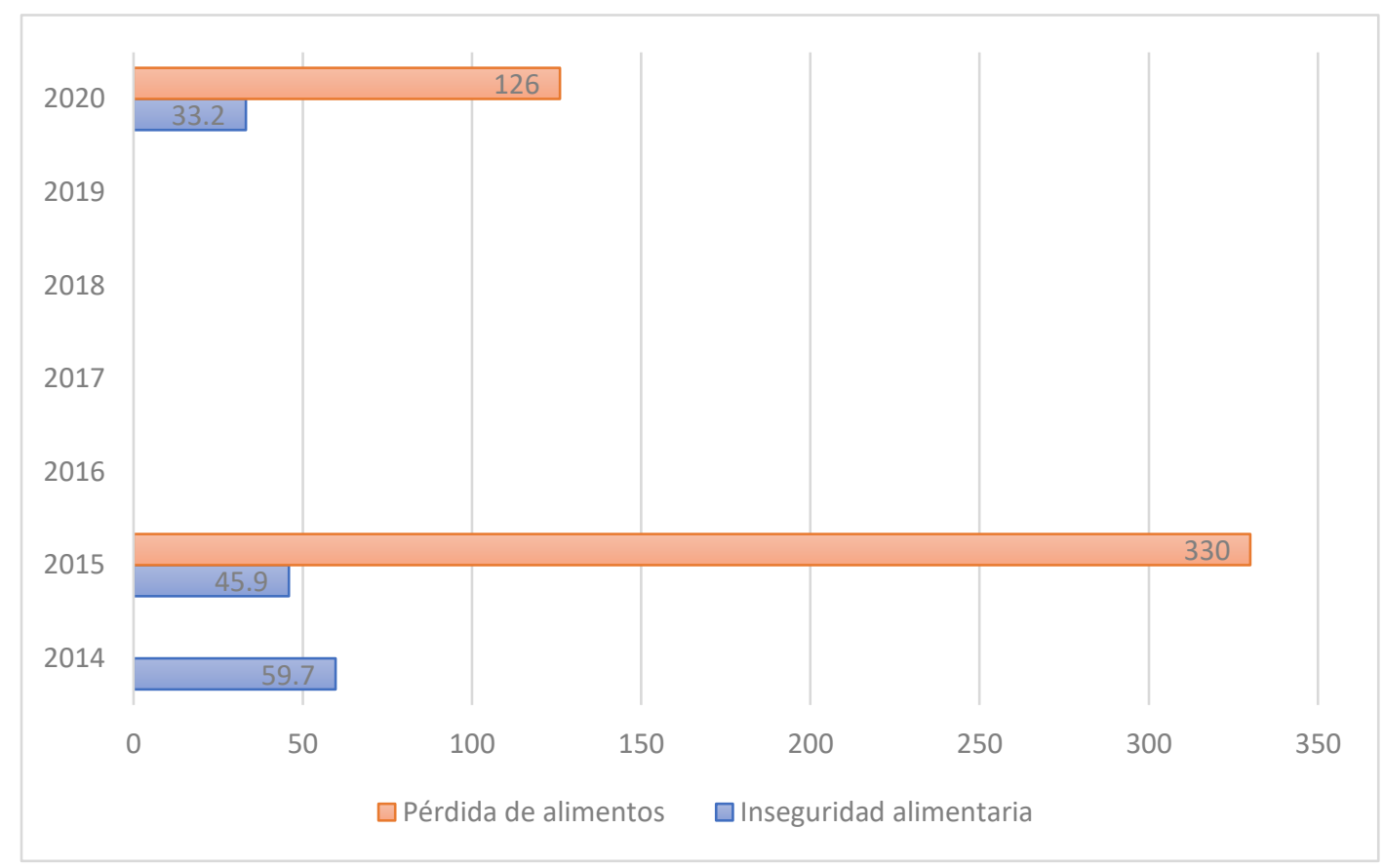

Elaboración propia. Fuente (FA0, 2020a)

Si bien en la región se evidencia, una creciente prevalencia de la inseguridad alimentaria desde el año 2014, la mitad de este incremento se dio entre 2019 y 2020 en el contexto de la pandemia. En cuanto a la pérdida de alimentos, es necesario resaltar que la mayor cantidad, se estima que ocurre en las primeras etapas de la cadena de producción principalmente en la recolección y distribución a mercados tanto mayoristas como minoristas. Lo que representa el $12 \%$ de pérdida de los alimentos destinados a consumo humano. La figura 1 muestra el impacto negativo que ha tenido la emergencia sanitaria, en cuanto a pérdida de alimentos en millones de toneladas y la prevalencia de la inseguridad alimentaria expresada en millones de personas impactadas.

Es necesario aclarar que, a la fecha de realización de este artículo, no se han actualizado cifras ni datos del impacto de la pandemia en cuanto a seguridad alimentaria y pérdida de alimentos. Sin embargo, se puede estimar que estos efectos adversos repercutirán negativamente en la economía, la biodiversidad, la soberanía alimentaria y a largo plazo la calidad de vida de la población de la región.

\section{Hacia la creación y optimización de acciones de mejora}

Para minimizar estos impactos es necesario seguir fortaleciendo las acciones integrales que han conseguido, de una parte, aprovechar las características ambientales y biodiversidad de la región. Estos favorecen la producción continua e involucran a todos los actores participantes en cada uno de los eslabones de la cadena agroalimentaria, para optimizar la gestión de residuos en origen hasta el consumo final.

Aunque en la región el interés por el uso productivo de los desechos es incipiente, el potencial de este es sin duda considerable si se toman en cuenta los volúmenes de producción de materias primas agrícolas y los 
desechos de las actividades agropecuarias, agroindustriales y antropogénicas. El uso de la biomasa de desecho puede sentar las bases del desarrollo de nuevas empresas y cadenas de valor de base biológica, con un fuerte énfasis en el uso de desechos, desarrolladas por jóvenes menores de 35 años, empresarios innovadores de la Región (Macías et al., 2020).

Por otra parte, diversos países han comenzado a fortalecer sus marcos de políticas pertinentes para la bioeconomía, entre ellas las de sostenibilidad e innovación en ámbitos relacionados. Tal es el caso de la agricultura y ganadería sostenible, el pago por servicios ambientales, las aplicaciones de la biotecnología, el aprovechamiento de la biodiversidad, los recursos forestales, el manejo de residuos y la producción de bioenergía (Rodríguez et al., 2017). Se evidencia un interés por el desarrollo de políticas que alienten iniciativas de agricultura familiar para el desarrollo local. Se trata de políticas más complejas e intersectoriales, mediante las cuales, con los mismos recursos, se intervienen varias dimensiones. Con estas políticas, la agricultura familiar sale de lo sectorial, se compromete en un sistema, se articula con nuevos actores, se fortalece en sus territorios y contribuye con el desarrollo sostenible. (Grisa \& Sabourin, 2019).

Estos retos suponen a los gobiernos de la región, el trabajo colaborativo interinstitucional y regional para la creación e implementación efectiva de políticas públicas. En este punto es de mencionar el Plan de Seguridad Alimentaria, Nutrición y Erradicación del Hambre de la CELAC 2025. Durante la III Cumbre de jefes de estado y de gobierno de la CELAC, que se llevó a cabo en Costa Rica en enero de 2015, los países participantes acogieron de manera formal esta hoja de ruta, la cual fue elaborada con el apoyo de la Comisión Económica para América Latina y el Caribe (CEPAL), la FAO y la Asociación Latinoamericana de Integración (ALADI).

El Plan se sustenta en cuatro pilares que buscan avanzar en la consecución de los ODS en la región, particularmente los objetivos 1, 2, 3, 6, 8, 12 y 13. Las líneas de trabajo de cada pilar corresponden al apoyo para la definición de marcos jurídicos de seguridad alimentaria, la mejora del comercio, la disminución de las pérdidas y desperdicios de alimentos. La optimización al acceso oportuno y sostenible a alimentos adecuados y suficientes para todas las personas, fomenta la mejora de los mercados de trabajo, especialmente el de la agricultura familiar. La optimización de la distribución de alimentos especialmente a los grupos vulnerables ante desastres de origen social y natural. La figura 2 muestra los pilares del Plan y su implicación en la consecución de los diferentes ODS mencionados.

Para la implementación de estas acciones es fundamental articular tres componentes: conocimiento e innovación, mediante el intercambio y gestión de conocimiento para la recolección de evidencias, el desarrollo tecnológico e innovación. Gobernanza y alianzas, a través de diálogos o redes de trabajo para fortalecer el marco normativo. Comunicación y sensibilización que busca la diseminación de mensajes que convoquen a todos los actores de los sistemas alimentarios en sus diferentes fases. 
Figura 2.

Articulación de ODS y líneas de acción del Plan de Seguridad Alimentaria, Nutrición y Erradicación del Hambre de la CELAC 2025.

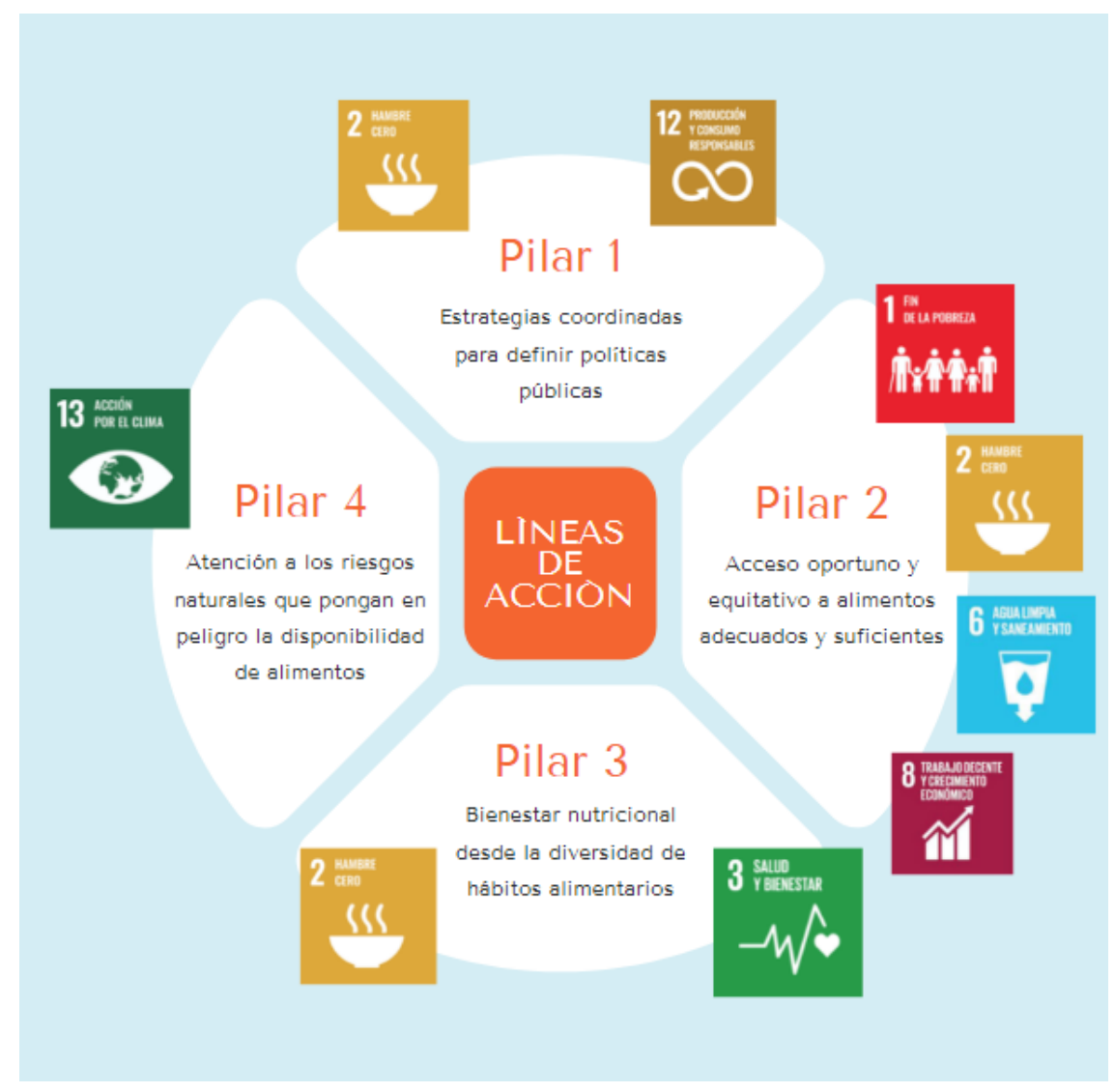

Elaboración propia. Fuente: Plan de Seguridad Alimentaria, Nutrición y Erradicación del Hambre de la CELAC 2025

Así se tiene que, dentro del ámbito de la acción para impulsar el fomento de la agricultura familiar, se destaca la implementación de los fondos concursables de FONTAGRO, que cuenta con el respaldo del Banco Interamericano de Desarrollo (BID) como representante legal y el Instituto Interamericano de Cooperación para la Agricultura (IICA). Mediante convocatorias para proyectos con duración de 36 meses, que demuestren innovación para sostenibilidad, inclusión, y rentabilidad, se promueve el trabajo colaborativo entre agricultores familiares que buscan mejorar su calidad de vida. Estas convocatorias público privadas, se han desarrollado con éxito en Bolivia, Chile, Costa Rica, Argentina y Paraguay, desde el año 2019. Es de resaltar que estas estrategias apoyan la creación de plataformas regionales de innovación que, por su cercanía con las características de cada zona geográfica, los intereses, necesidades de los grupos humanos locales, pueden asegurar el éxito de los proyectos beneficiados. FONTAGRO cofinancia iniciativas que generen bienes públicos regionales (BPR) agropecuarios. Donde los países comparten desafíos y oportunidades de crecimiento y desarrollo que son atendidos más eficientemente cuando las instituciones trabajan en forma colectiva, participativa y cooperativa. En ese sentido, las plataformas regionales promovidas por FONTAGRO son, en sí mismas, un BPR, como así también el conocimiento y las lecciones aprendidas que ellas generan (FONTAGRO, 2021). 
Otras acciones más localizadas, también consiguen coordinar estrategias contra la pérdida y desperdicio alimentario y respaldan el trabajo colaborativo de los actores de la cadena alimentaria. La academia como uno de estos actores, ha sido clave para garantizar el buen funcionamiento de los planes trazados. Varias de estas iniciativas se forjaron por el compromiso decidido de grupos de activistas que reivindican el crecimiento productivo a la par de la calidad de los alimentos, la soberanía y el acceso universal a ellos, replanteando un consumo más sostenible y consciente.

Estos fenómenos se han venido dando desde la década de los 70, como resultado de una búsqueda hacia la definición de este tipo de consumidores. Se pretende contribuir al desarrollo de una economía social y solidaria, regida por los valores de la cooperación, democracia, ciudadanía global, igualdad, solidaridad y medioambiente. Donde las relaciones comerciales se apoyan en los principios y valores del comercio justo de un mercado social, de una economía global y solidaria, en línea con los Objetivos de Desarrollo Sostenibles (Latorre, 2018).

Gracias al rápido desarrollo de las nuevas tecnologías de la información, es innegable la interconexión inmediata a la que están abocadas las generaciones actuales, por medio de ellas, han conseguido hacerse eco de manifestaciones sociales y culturales de nivel global. Tal es el caso del movimiento internacional "Slow Food", nacido en el año 1980 en Italia, gracias al interés de un grupo de activistas motivados a resguardar valores tradicionales de la buena alimentación dentro del paradigma de vida lento. Una de sus principales acciones es el "Disco Sopa". Estas experiencias tienen como común denominador la decidida participación de jóvenes de todo el mundo, quienes a pesar de las diferencias culturales han conseguido abanderar la valorización de los residuos agrícolas y del potencial de sus productos y subproductos, que impactan directamente en la dinamización de la economía local, mediante la creación de nuevos mercados y la generación de fuentes de empleo.

\section{Buenas prácticas y experiencias motivadoras desde la academia contra pérdida y despilfarro alimentario en el mundo}

En los últimos 10 años en América Latina y El Caribe, se han venido sumando movimientos asociativos y colaborativos que, con propósitos similares, cuestionan el paradigma de sistema económico en el que vivimos y reivindican la necesidad de fortalecer un cambio holístico hacia la sostenibilidad.

Merece entonces realizar una revisión sobre la implicación de los jóvenes en este tipo de movimientos socio económicos, que de manera transversal tienen como objetivo la lucha contra el cambio climático y la mejora de la seguridad alimentaria. Sin embargo, la presente reflexión se ocupará de explorar el papel fundamental de la academia, que, trascendiendo su responsabilidad social, debe considerar su esencia como gestora de transferencia de conocimiento y dinamizadora del proceso de formación de los nuevos jóvenes profesionales, como ciudadanos participativos en los cambios económicos, políticos, sociales y culturales del desarrollo sostenible a nivel global. La educación es considerada la herramienta más poderosa que puede cohesionar las inquietudes y pesadumbres económicas, sociales y ambientales bajo una estrategia de desarrollo sostenible. De esto se deriva que la responsabilidad social, debe impregnar las funciones y las prácticas de las universidades (Chen \& Nasongkhla, 2019). 
Varias instituciones de educación superior en el mundo, se han sumado a la lucha contra despilfarro alimentario desde diferentes enfoques, política pública, salud, mercadotecnia, antropología, negocios sostenibles, todas ellas interrelacionadas y articuladas (Tapia \& López, 2020). Para mencionar algunas de estas actividades de cada continente, se puede citar la red virtual de formación y aprendizaje desarrollada por el Institute of Industrial Environment Economics de la Universidad de Lund de Suecia y dirigida a adolescentes de 16 a 18 años de institutos de secundaria de todo el mundo, quienes reciben formación de manera gratuita, sobre concienciación social, ambiental y económica; desde su lanzamiento en 1999 han participado más de 25.000 adolescentes.

La Universidad de Otago, Nueva Zelanda, apuesta por fortalecer la investigación y la transferencia de conocimiento, desde uno de sus departamentos especializados en la innovación contra el desperdicio de alimentos. El cual reúne investigadores senior, cuyos productos académicos soportan los lineamientos gubernamentales, las políticas curriculares de las instituciones de formación y educación superior de Asia Pacífico. Se desarrollan como temáticas centrales la innovación social y comportamiento del consumidor, gestión e indicadores de pérdida, desperdicio y residuos alimentarios e innovaciones tecnológicas. Igualmente, estas líneas están conformadas por investigadores asociados, profesores titulares que guían a estudiantes en prácticas empresariales, para reconocer las necesidades del sector de la gastronomía y restauración (Grisa \& Sabourin, 2019). Además de ofrecer propuestas de mejora y personal administrativo de áreas de la responsabilidad social corporativa, que se encargan de hacer operativos los hallazgos de los productos de investigación.

A nivel de Iberoamérica, es de resaltar el Observatorio del Derecho a la Alimentación, con el apoyo de la Agencia Española de Cooperación Internacional para el Desarrollo AECID, ochenta universidades de más de 17 países conforman esta red, unidos por el propósito común de favorecer el intercambio de conocimiento sobre seguridad alimentaria y nutrición. En la última cumbre celebrada de manera presencial, el 17 de octubre de 2019 y bajo el hashtag \#UniversidadesContraEIHambre, se llegaron a consensos y compromisos enmarcados dentro del plan Interconecta. El cual favorece la transferencia, intercambio y gestión de conocimiento para el desarrollo, enfocándose en temáticas como seguimiento de indicadores del derecho humano a la alimentación adecuada (DHAA), agendas y políticas públicas para reducir los fenómenos de sobrepeso y obesidad, pérdidas y desperdicios de alimentos, entre otros. Para la comunicación de resultados y buenas prácticas, se propuso fortalecer mecanismos de intercambios virtuales, los cuales se han visto catapultados en el mundo entero, por el desarrollo de tecnologías, en los últimos dos años.

Otro claro ejemplo de construcción de alianzas es el centro de los objetivos de desarrollo sostenible para América Latina, como parte de la red de Soluciones Sostenibles de Naciones Unidas (SDSN), vinculado con el Earth Institute de la Universidad de Columbia, el CODS-LAC, tiene su sede en la Universidad de los Andes y cuenta con la participación activa de la Universidad Javeriana y Universidad del Norte y el Centro Internacional de Agricultura Tropical (CIAT) de Colombia, Universidad Católica de Chile, Universidad Autónoma de México y TEC de Monterrey de México, Universidad del Pacífico de Perú y Universidad de Campiñas de Brasil.

Desde su constitución, el CODS ha venido generando espacios de encuentro para el debate sobre la implementación de los objetivos de desarrollo sostenible con instituciones de educación superior de América Latina y el Caribe. Con el objetivo implícito de visibilizar las características y necesidades del continente como una región de gran biodiversidad, pero también con condiciones de alta inequidad o violencia. Esto lo consigue 
mediante seminarios y convocatorias de investigación en los que se socializan resultados de productos académicos con propuestas para resolver retos de la región, la cátedra abierta un espacio de debates con temáticas específicas, cursos en línea abiertos desde la plataforma Coursera dirigida a público en general y una versión específica para empresarios, con el propósito de brindar herramientas con las que puedan aportar a la agenda 2030 (Zhong et al., 2018).

Particularmente sobre la merma de pérdida y desperdicio alimentario, correspondiente al ODS 2 hambre cero, ODS 12 producción y consumo responsables y ODS 17 alianzas para lograr los objetivos. Este centro ha llevado a buen término varias convocatorias con la entrega de capital semilla, para asegurar la continuidad en investigación de la producción de alimentos sostenibles y la merma de despilfarro alimentario (De los Ríos, 2018). Se priorizan las investigaciones de equipos interculturales e interdisciplinarios que generen nuevas perspectivas desde los sistemas ambientales, económicos y sociales, que tengan en cuenta componentes de gobernanza ambiental e instrumentos de política, sistemas agroalimentarios sostenibles y la mejora de calidad de vida de pequeños agricultores y productores.

Dentro del fortalecimiento del trabajo multidisciplinario e intercultural entre investigadores de América Latina y el Caribe, el CODS promueve la inserción de las recomendaciones de política pública, para que la replicabilidad, escalabilidad y aplicación del trabajo investigativo, logre llegar de una manera rápida y efectiva a las poblaciones más vulnerables. Por último, como muestra fehaciente de la innovación tecnológica y la consecución de alianzas, se creó el CODspace, para promover el uso de las imágenes satelitales del Programa Copernicus de la Agencia Espacial Europea desde el laboratorio de innovación de la Facultad de Ingeniería de la Universidad de Los Andes. La cual busca entre otros objetivos, obtener datos concretos para monitorear en tiempo real, avances o retrocesos de los ODS, todo ello mediante sensores remotos y algoritmos de inteligencia artificial.

De acuerdo con la FAO (2019)la pérdida de alimentos se refiere a la disminución de la masa de alimentos comestible específicamente para consumo humano, que se produce durante las etapas de producción, post cosecha, procesamiento, almacenamiento, transporte y distribución. Mientras que el desperdicio alimentario son los descartes en las etapas finales de las cadenas durante la venta minorista o retail hasta el consumo en los hogares y servicios de alimentación (Meybeck et al., 2012).

Frente a esta problemática surgen propuestas como la de la ONG CCORI, cocina óptima, una propuesta liderada por el chef Palmiro Ocampo, quien promueve la revalorización de desperdicios alimentarios en su país natal Perú. Este concepto hace referencia a reaprovechar y encontrar valor no solo nutricional sino también económico, a aquellos productos alimentarios de consumo humano, que se encuentran en perfecto estado, y que no son finalmente aprovechados por fallas en la cadena de producción de alimentos. Por ejemplo, frutas golpeadas o que no cumplen con los cánones de estética para ser comercializados. En CCORI se hacen presentes las alianzas como centro articulador de saberes. Mediante talleres, charlas y tanques de pensamiento dirigidos a estudiantes y población vulnerable, diferentes organismos del sector público de Perú y centros universitarios como la Universidad San Ignacio de Loyola, han conseguido sensibilizar y transmitir buenas prácticas a esta población.

Igualmente, la Universidad de Lima junto con el IDIIC (Instituto de Investigación Científica) presentó en la primera edición del Functional Food Expo "Valorando la Biodiversidad Peruana" del 2019, una bebida 
multifuncional bebida, fuente de omega 3 y antioxidantes elaborada con alimentos nativos como el camu camu y el mango, una buena alternativa de aprovechamiento que propende por una dieta saludable y el desarrollo de la industria agro alimentaria de un país megadiverso como el Perú.

\section{CONCLUSIONES}

Para hacer frente a los continuos y cada vez más rápidos cambios que enfrenta el continente latinoamericano en cuanto a seguridad alimentaria, pérdida y desperdicio de alimentos, es primordial articular esfuerzos y acciones efectivas desde el gobierno, sector privado y la población, que estén encausados a desarrollar una visión y soluciones comunes.

En lo que respecta a la academia, como actores decisivos de liderazgo social, pueden y deben materializar acciones transversales, tendientes a fortalecer el desarrollo sostenible de la región. Desde las buenas y exitosas prácticas revisadas, se revelan estrategias que se han soportado en una estructura orgánica bien definida, la cual garantiza la optimización de procesos y de creación de espacios de discusión, debate y toma de decisiones, como unidades de pensamiento y acción. Particularmente para generar cambios estructurales en su comunidad académica hacia la prevención de pérdida y desperdicio alimentario, se hace indispensable la labor de motivarla mediante planes de comunicación adaptados a las necesidades de directivas, docentes, estudiantes y administrativos. Los cuales ayudan a generar la adquisición de competencias de producción y consumo responsable, pensamiento crítico sobre los desafíos actuales de la región, habilidades en nuevas tecnologías e innovación, la construcción de alianzas estables y duraderas, entre otras acciones.

Respecto a la selección y diseño de las estrategias institucionales, se pueden identificar prácticas comunes en los casos de éxito mencionados en el anterior apartado, como la definición de unidades académicas y administrativas que demuestren voluntad. La infraestructura mínima necesaria, para articular su saber hacer con otros departamentos implicados, reconocer áreas de interés compartido para determinar objetivos comunes y espacios de colaboración, así como fortalezas y oportunidades de mejora a nivel institucional y por áreas de conocimiento.

\section{Conflicto de intereses / Competing interests:}

La autora declara que no incurre en conflictos de intereses.

\section{Rol de los autores / Authors Roles:}

No aplica

\section{Fuentes de financiamiento / Funding:}

La autora declara que no recibió un fondo específico para esta investigación.

\section{Aspectos éticos / legales; Ethics / legals:}

La autora declara no haber incurrido en aspectos antiéticos, ni haber omitido aspectos legales en la realización de la investigación.

\section{REFERENCIAS}

Bélanger, J., \& Pilling, D. (2019). The State of the World's Biodiversity for Food and Agriculture. FA0. https://doi.org/10.4060/CA3129EN

Chen, S., \& Nasongkhla, J. (2019). Discover the new Right to education handbook. Unesco. 
https://en.unesco.org/news/discover-new-right-education-handbook

De los Ríos, I. (2018). Cátedra banco de alimentos de UPM Memoria Anual de Actividad. https://cutt.ly/3mIGPPj FA0. (2019). Más de 820 millones de personas pasan hambre y unos 2000 millones sufren su amenaza. Noticias ONU. https://news.un.org/es/story/2019/07/1459231

FAO. (2020a). Seguridad Alimentaria bajo la Pandemia de COVID-19. Organización de las Naciones Unidas para la Alimentación y la Agricultura. https://www.fao.org/family-farming/detail/es/c/1287511/

FA0. (2020b). Sistemas alimentarios y COVID-19 en América Latina y el Caribe No 9: cómo disminuir las pérdidas y desperdicios de alimentos. CEPAL, FAO. https://www.cepal.org/es/publicaciones/45768sistemas-alimentarios-covid-19-america-latina-caribe-ndeg-9-como-disminuir

FONTAGRO. (2021). Aumento de la productividad en la agricultura familiar con sostenibilidad, inclusión, y rentabilidad. https://www.fontagro.org/type/convocatoria-2019-productividad/

Grisa, C., \& Sabourin, E. (2019). Agricultura Familiar: de los conceptos a las políticas públicas en América Latina y el Caribe. FA0. https://www.fao.org/documents/card/es/c/ca5087es/

Latorre, J. (2018). Economía social y solidaria. https://www.uahurtado.cl/centrovives/economia-social-ysolidaria/

Macías, M., Girón, C., Nieto, M., Chavrier, N., Páez, D., Ureña, M., Moreno, J., García, M., \& de la Viña, G. (2020). Tecnologías de bioeconomía para valorizar residuos y desperdicios: oportunidades de negocio para la agricultura familiar [Instituto Interamericano de Cooperación para la Agricultura (IICA)]. https://repositorio.iica.int/handle/11324/12942

Meybeck, A., Burlingame, B., Dernini, S., \& Gitz, V. (2012). Improving food systems for sustainable diets in a Green economy. https://doi.org/10.13140/RG.2.2.22093.90083

Rodríguez, A., Mondaini, A., \& Hitschfeld, M. (2017). Bioeconomía en América Latina y el Caribe: contexto global y regional y perspectivas. CEPAL. https://www.cepal.org/es/publicaciones/42427-bioeconomiaamerica-latina-caribe-contexto-global-regional-perspectivas

Tapia, M., \& López, S. (2020). Bancos de alimentos. un modelo que funciona mundialmente en la lucha contra el hambre. Agroalimentaria. Revista agroalimentaria, 26(50), 167-182. https://dialnet.unirioja.es/servlet/articulo?codigo=7782790

Zhong, T., Si, Z., Crush, J., Xu, Z., Huang, X., Scott, S., Tang, S., \& Zhang, X. (2018). The impact of proximity to wet markets and supermarkets on Household dietary diversity in Nanjing City, China. Sustainability, 10(5), 1465. https://doi.org/10.3390/su10051465 